\title{
The potential role of microRNAs in regulating gonadal sex differentiation in the chicken embryo
}

\author{
Andrew D. Cutting • Stephanie C. Bannister • \\ Tim J. Doran • Andrew H. Sinclair • \\ Mark V. L. Tizard • Craig A. Smith
}

Published online: 13 December 2011

(C) Springer Science+Business Media B.V. 2011

\begin{abstract}
Differential gene expression regulates tissue morphogenesis. The embryonic gonad is a good example, where the developmental decision to become an ovary or testis is governed by female- or male-specific gene expression. A number of genes have now been identified that control gonadal sex differentiation. However, the potential role of microRNAs (miRNAs) in ovarian and testicular pathways is unknown. In this review, we summarise our current understanding of gonadal differentiation and the possible involvement of miRNAs, using the chicken embryo as a model system. Chickens and other birds have a ZZ/ZW sex chromosome system, in which the female, $\mathrm{ZW}$, is the heterogametic sex, and the male, $\mathrm{ZZ}$, is homogametic (opposite to mammals). The Z-linked DMRT1 gene is
\end{abstract}

Responsible Editors: Tariq Ezaz and Jennifer Graves.

A. D. Cutting · T. J. Doran · M. V. L. Tizard

CSIRO Australian Animal Health Laboratories,

Portarlington Rd,

Geelong, Victoria, Australia

A. D. Cutting - T. J. Doran - A. H. Sinclair ·

C. A. Smith $(\bowtie)$

Murdoch Childrens Research Institute,

Royal Children's Hospital,

Parkville, Victoria 3052, Australia

e-mail: craig.smith@mcri.edu.au

A. D. Cutting - A. H. Sinclair - C. A. Smith

Poultry Cooperative Research Centre,

Armidale, NSW, Australia thought to direct testis differentiation during embryonic life via a dosage-based mechanism. The conserved SOX9 gene is also likely to play a key role in testis formation. No master ovary determinant has yet been defined, but the autosomal FOXL2 and Aromatase genes are considered central. No miRNAs have been definitively shown to play a role in embryonic gonadal development in chickens or any other vertebrate species. Using next generation sequencing, we carried out an expression-based screen for miRNAs expressed in embryonic chicken gonads at the time of sexual differentiation. A number of miRNAs were identified, including several that showed sexually dimorphic expression. We validated a subset of miRNAs by qRT-PCR, and prediction algorithms were used to identify potential targets.

S. C. Bannister

Max F. Perutz Laboratories GmbH (MFPL),

Dr. Bohr-Gasse,

91030 Vienna, Austria

C. A. Smith

Department of Zoology, The University of Melbourne, Melbourne, Victoria, Australia

A. D. Cutting - A. H. Sinclair · C. A. Smith Department of Paediatrics, The University of Melbourne, Melbourne, Victoria, Australia 
We discuss the possible roles for these miRNAs in gonadal development and how these roles might be tested in the avian model.

Keywords Testis · ovary · TGF-beta signalling · miRNA $\cdot$ AMH $\cdot$ Gonad $\cdot$ Sex determination

\begin{tabular}{|c|c|}
\hline \multicolumn{2}{|c|}{ Abbreviations } \\
\hline ACVR1A & Activin A receptor type IA \\
\hline $\mathrm{AMH}$ & Anti-Müllerian hormone \\
\hline AMHR2 & AMH receptor 2 \\
\hline BMPR1A/B & $\begin{array}{l}\text { Bone morphogenetic protein } \\
\text { receptor type IA/B }\end{array}$ \\
\hline DMRT1 & $\begin{array}{l}\text { Doublesex and mab-3-related } \\
\text { transcription factor } 1\end{array}$ \\
\hline FOXL2 & Forkhead box L2 \\
\hline Gga-mir- & Gallus gallus microRNA \\
\hline $\mathrm{HH}$ & $\begin{array}{l}\text { Hamilton Hamburger chicken } \\
\text { embryonic stage }\end{array}$ \\
\hline miRISC & miRNA loaded RISC complex \\
\hline miRNA & microRNA \\
\hline RISC & RNA-induced silencing complex \\
\hline RSPO1 & $\begin{array}{l}\text { R-spondin homolog } \\
\text { (Xenopus laevis) } 1\end{array}$ \\
\hline SMAD & $\begin{array}{l}\text { Mothers Against Decapentaplegic } \\
\text { homolog }\end{array}$ \\
\hline SOX9 & SRY (Sex-determining Region Y)-box 9 \\
\hline SRY & Sex-determining Region Y \\
\hline TGF- $\beta$ & Transforming growth factor beta \\
\hline TGIF & TGF- $\beta$-induced factor homeobox \\
\hline Wnt4 & $\begin{array}{l}\text { Wingless-type MMTV integration } \\
\text { site family member } 4\end{array}$ \\
\hline ZEB1/2 & Zinc finger E-box binding homeobox \\
\hline
\end{tabular}

\section{Introduction}

In higher vertebrates, the male and female sexes exhibit physiological and behavioural differences required for sexual reproduction. These differences are the result of two processes occurring during embryonic development, sex determination and sexual differentiation. The former is a decision as to what sex the organism will become, the latter being the development of a sexspecific phenotype. In organisms where sex is genetically determined, the combination of sex chromosomes at fertilisation determines sex. Sexual differentiation occurs later and is traditionally thought to start with development of the embryonic gonads into testes or ovaries. The gonads then secret masculinising or feminising hormones that initiate sex-specific development. However, recent studies have challenged this view, providing evidence that sexual differentiation may be cell autonomous, at least in birds, and that the sexual phenotype is at least partly determined on a cell-by-cell basis (Zhao et al. 2010). Nevertheless, gonadal sex differentiation (testis versus ovary formation) is a key aspect of sexual development.

Recent years have seen some major advances in our understanding of the molecular genetics underlying gonadal sex differentiation, in the chicken and in other vertebrates (Koopman 2001; Morrish and Sinclair 2002; MacLaughlin and Donahoe 2004; Smith and Sinclair 2004; Wilhelm et al. 2007; Graves 2009; Sekido and Lovell-Badge 2009; Sekido 2010; Smith 2010; Chue and Smith 2011). Though many genes co-ordinating gonad development have been discovered, regulatory interactions amongst these genes are less clear. One area of growing interest in the field of reproduction and sexual differentiation is the likely involvement of small non-coding RNAs, especially microRNAs (miRNAs). MiRNAs are known to regulate cell division and cell fate and identity (reviewed, Pauli et al. 2011; Suh and Blelloch 2011). Furthermore, miRNAs have been detected in mammalian and avian gonads during development (Bannister et al. 2009; Huang et al. 2010; Tripurani et al. 2010; Torley et al. 2011). Some of these gonadal miRNAs show sexually dimorphic expression patterns and are candidate regulators of sex-specific development. Here, we review how miRNAs may be involved in embryonic gonad development using the chicken embryo as a model system.

\section{Gonadal development in the chicken}

The embryonic gonads develop in a similar way in mammals and birds, involving conserved cell types and developmental processes that are generally shared (Lovell-Badge et al. 2002; Sekido and Lovell-Badge 2007; Smith 2007; Chue and Smith 2011). This implies that the underlying genetic pathways leading to testis or ovary formation are also conserved. Indeed, the chicken shares with other vertebrates several key genes involved in gonadal sex differentiation, such as DMRT1, SF1, SOX9 and AMH, as outlined below (Smith and Sinclair 2004). The chicken therefore 
provides a practical model for studying embryonic gonad development (Morrish and Sinclair 2002; DeFalco and Capel 2009). In the chicken, sex is determined genetically: male is the homogametic sex (ZZ) and female is heterogametic (ZW). In both sexes, embryonic gonads appear on the ventral surface of mesonephric kidneys at embryonic day (E) 3.5 (Fig. 1). This is equivalent to Hamburger and Hamilton (HH) stage 19-20, (Hamburger and Hamilton 1951). Gonads at this stage comprise an inner region, the medulla, surrounded by a thin outer cortex. The germ cells, having migrated via the bloodstream from the extra-embryonic germinal crescent, settle in the gonads prior to sexual differentiation (E3-4). The gonads are histologically identical between the sexes at this time (up to day E5; HH stage 28). Gonadal sex differentiation commences at E6-6.5 (HH stage 29-30) and marks the first point at which sex differences in the gonad are histologically detectable. In males (ZZ), the first morphological sign of testicular differentiation is the condensation of pre-Sertoli cells within the medulla of the gonads. These pre-Sertoli cells aggregate into presumptive seminiferous cords. During this time, germ cells migrate into the cords, and steroidogenic "interstitial" cells later differentiate around the cords (Martineau et al. 1997; Smith et al. 2005). The outer layer of cells (the cortex) subsequently reduces to an epithelial monolayer. In females (ZW), ovarian differentiation begins with a thickening of the cortical layer. However, only the left gonad develops into a functional ovary. The right gonad regresses and becomes rudimentary. The ovarian cortex grows and accumulates germ cells, while the medulla of both left and right gonads becomes fragmented and develops fluid-filled cavities called lacunae (Fig. 1).

\section{Molecular control of gonadal differentiation in the chicken embryo}

Testis

In mammals, the Y chromosome-linked $S R Y$ gene (Sex-determining Region $\underline{Y}$ ) initiates testis differentiation in males $(\mathrm{XY})$. There is no SRY homolog in the chicken, and a master sex-determining gene has not yet been identified. However, DMRT1 (Doublesex and mab-3-related Transcription factor 1 ) is required for testis differentiation in the chicken. DMRT1 is Z-linked and is exclusively expressed in the urogenital system in chicken embryos. It is more highly expressed in males, and its knockdown leads to feminisation of ZZ gonads (Smith et al. 2003, 2009). It has been suggested that DMRT1 in males may activate SOX9 (SRY (Sex determining region Y)-box 9) (Smith et al. 2009; Chue and Smith 2011), which is required for Sertoli cell differentiation during testis development in mammals (Kobayashi 2005) (Fig. 2). Although the knockdown of DMRT1 reduces testicular SOXO expression (Smith et al. 2009), expression of the two genes is not temporally aligned. Furthermore, DMRT1 is also expressed in

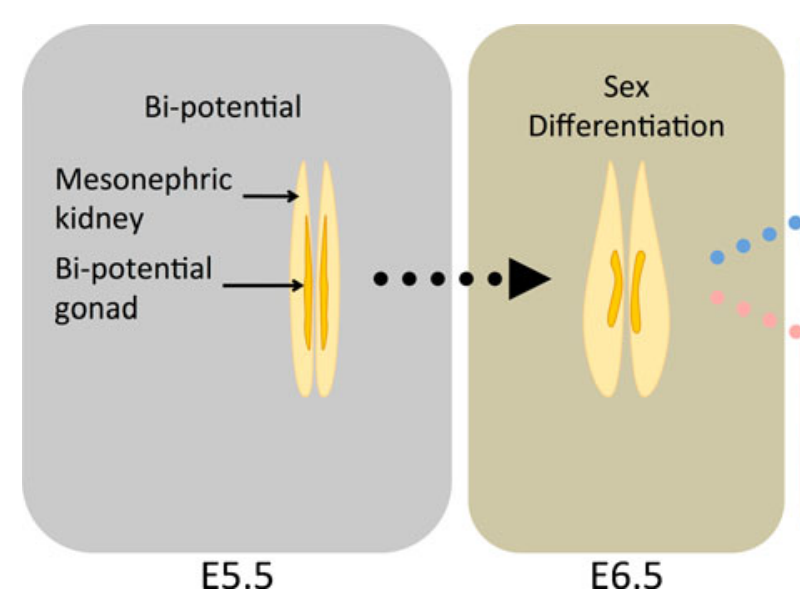

Fig. 1 Sexual differentiation of the embryonic gonad in the chicken. Gonads appear ventral to the mesonephric kidney and remain bi-potential until embryonic day (E) 6.5, when morphological differences develop. By E9.5, the medulla of the testis

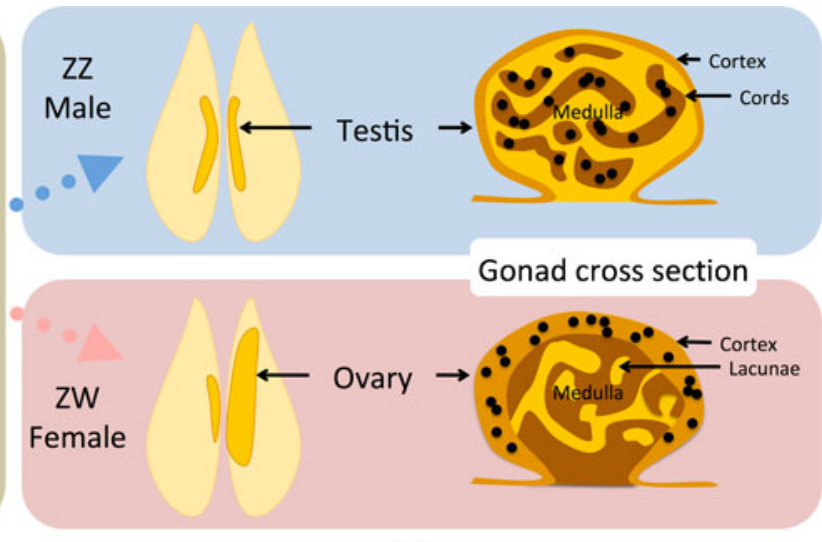

E9.5

(ZZ) has well-developed cord structures that are populated by PGCs (dot), whereas in the ovary (ZW), PGCs populate the cortex, which is now thickened 


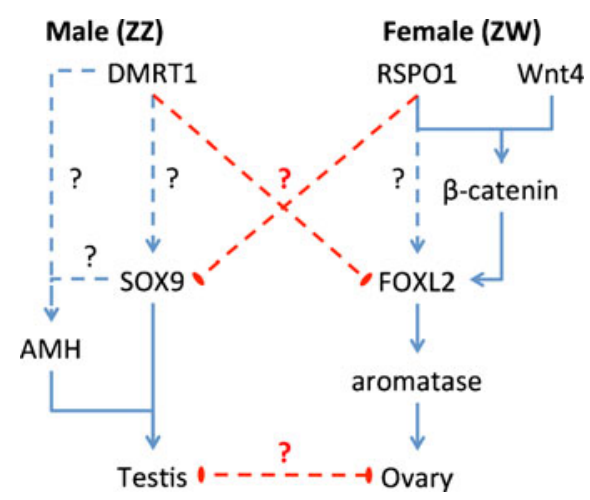

Fig. 2 Key genes involved in chicken gonadal sex differentiation based on expression profiling and knockdown analysis. In males (ZZ), DMRT1 is likely to indirectly activate $S O X 9$ expression, which is critical for testis differentiation. In females (ZW), RSPO1 leads to activation of the $\mathrm{Wnt} 4 / \beta$-catenin pathway, and together with FOXL2/aromatase leads to ovary differentiation. DMRT1 and FOXL2 may act to antagonise the ovarian and testicular differentiation pathways, respectively, as occurs in mammals

females, albeit at lower levels (Oreal et al. 2002; Koba et al. 2008), but $S O X 9$ is not expressed in female gonads. If DMRT1 does indeed regulate $S O X 9$, it is likely that other male-specific factors intervene.

In male mammals, embryonic Anti-Müllerian Hormone (AMH) is expressed in Sertoli cells and functions to regress the Müllerian ducts, which would otherwise form the uterus and Fallopian tubes (Rey et al. 2003). Mammalian females express very little if any $\mathrm{AMH}$ during gonadal development, which allows the Müllerian ducts to develop into the internal female genitalia. Contrary to mammals, chicken $A M H$ is expressed in both sexes at low levels but is upregulated in males specifically during gonadal differentiation (Oreal et al. 1998; Oreal et al. 2002; Koba et al. 2008). As in mammals, AMH is thought to trigger the disintegration of Müllerian ducts in male chicken embryos. The right duct also disintegrates in female chicken embryos, which may explain the expression of $A M H$ in ZW embryos (the left duct of females forms a functional oviduct, and it is thought that the left duct is protected from AMH by local oestrogen production). In mammals, Sox9 participates in the activation of $\mathrm{Amh}$ gene expression in Sertoli cells (De Santa Barbara et al. 1998; Lasala et al. 2011) (Fig. 2). However, chicken $A M H$ expression precedes that of SOX9 (Oreal et al. 1998), at least at the mRNA level, suggesting that its activation is not dependent upon SOX9. Interestingly, male-to-female sex reversal, including Müllerian duct regression, can be induced by grafting a late stage embryonic testis to the vasculature of female chicken embryos prior to ovarian differentiation (Frankenhuis and Kappert 1980; Maraud et al. 1990; Rashedi et al. 1990). The likely factor inducing sex reversal in this instance is $\mathrm{AMH}$, which may have a more central role in avian testis formation than it does in mammals.

Ovary

In the mammalian embryo, two important ovarian pathways have been defined. The first involves the canonical $\beta$-catenin signalling pathway. In this pathway, ovarian signalling molecules R-Spondin 1 (Rspo1) and Wnt-4 activate the $\beta$-catenin pathway in the developing female gonad (Fig. 2). Rspo1 is currently thought to activate Wnt 4 , and they then act together to stabilise $\beta$-catenin (Tomizuka et al. 2008). XX $\beta$-catenin null mice develop masculinised gonads, and this effect is very similar in mice with targeted deletions of Rspol or Wnt4 (Liu et al. 2009). Therefore, the $\beta$-catenin pathway represents a critically important regulator of ovarian development, at least in mammals. The same may also apply to birds.

The second pathway leading to ovarian development involves the transcription factor FOXL2 (forkhead box (winged helix)). In mammals, FOXL2 activates key events involved in ovarian growth and differentiation, such as aromatase enzyme expression, inhibin and follistatin gene expression, and granulosa cell development (Harris et al. 2002; Schmidt et al. 2004; Blount et al. 2009). In the chicken, FOXL2 is also activated femalespecifically at the time of sexual differentiation (E5.0; HH stages 27-28), and the protein co-localises with aromatase enzyme in medullary cells of the developing ovary (Govoroun et al. 2004; Hudson et al. 2005). Aromatase converts androgens to oestrogens, and is likely to be activated by FOXL2 (Govoroun et al. 2004; Hudson et al. 2005; Fleming et al. 2010). Oestrogens are potent feminising factors in non-mammalian vertebrates. Avian males treated with oestrogen can develop transient ovaries (reviewed Scheib 1983), while inhibition of aromatase enzyme activity can induce sex reversal of female chicken embryos (Elbrecht and Smith 1992; Vaillant et al. 2001). Aromatase therefore represents a critical factor required for gonadal sex differentiation of the chicken, indicating that steroid hormones play crucial roles in the early stages of avian gonad development. However, neither the aromatase gene nor its potential activator, FOXL2, is sex linked in the 
chicken. The upstream activator of this FOXL2aromatase pathway in ZW embryos is currently unknown.

It has been proposed that the male and female differentiation pathways are mutually antagonistic, both at the embryonic and postnatal stages (Kim et al. 2006; Sinclair and Smith 2009; Veitia 2010). For example, in the mouse embryo, Sox9 and Wnt4 mutually antagonise each other during testis and ovarian formation (Barske and Capel 2008). Ablation of critical gonadal sex-determining factors at postnatal stages can cause transdifferentiation of the gonad, and development of characteristics of the opposite sex. For example, ablation of FOXL2 in postnatal female mice results in testis-like cord structures that express SOX9 and AMH and harbour differentiated spermatogonia (Uhlenhaut et al. 2009). Similarly, deletion of DMRT1 in postnatal mice allows reprogramming of Sertoli cells to granulosa cells that express FOXL2 (Matson et al. 2011). These studies not only demonstrate the lability of supposedly terminally differentiated gonads, but they also show that the sexual phenotype of differentiated gonads needs to be constantly maintained in a mutually antagonistic environment (Fig. 2). Whether this post-embryonic antagonism in mammals also prevails in the chicken system is unknown.

Of particular interest is the regulation of genes that are expressed in both sexes but at different levels. For example, chicken DMRT1 and $A M H$ are expressed in the gonads of both sexes but more highly in males. How is this differential expression regulated? Regulation could occur at the transcriptional level, with a different set of factors operating in males versus females. An alternative possibility is post-transcriptional regulation. In this context, a potential role exists for regulatory control by miRNAs. We and others have detected miRNAs in embryonic gonads, where they may modulate the genetic pathways required for sexual differentiation (Bannister et al. 2009; Hossain et al. 2009; Huang et al. 2010; Torley et al. 2011; Tripurani et al. 2010).

\section{MiRNA biogenesis and function}

MiRNAs are post-transcriptional regulators of gene expression, which act by negatively regulating specific target mRNAs. MiRNA genes are located within intragenic or intergenic regions and can be autonomously regulated or regulated together with a host gene transcript. The miRNA forms a characteristic hairpin structure within the primary transcript. This is recognised by the Drosha and Dicer endonucleases that produce the mature 21-23 nt miRNA (summarised in Fig. 3). The mature miRNA is then loaded into the RNA-induced silencing complex (RISC) to form the activated miRNA-RISC complex (miRISC). RISC is the chief regulating component in this process, whereas the miRNA simply establishes RISC specificity to target mRNAs. Though most functional target sites for miRNAs have been identified in the $3^{\prime}$ untranslated regions (UTRs) of mRNAs, growing evidence suggests that functional sites may also exist in the 5' UTR and coding regions of mRNAs (Easow et al. 2007; Lytle et al. 2007; Duursma et al. 2008; Forman et al. 2008). RISC is primarily associated with translational repression, and it does so by a number of means, including mRNA degradation or destabilisation by slicing or de-adenylation, interfering with ribosomal assembly and progression, or degradation of polypeptide synthesis via proteinase recruitment. Less commonly, miRNAs may also upregulate translation (Vasudevan et al. 2007) or translocate mRNAs to $\mathrm{p}$ bodies (Liu et al. 2005), presumably storing the transcript for future translation or degradation. For in-depth reviews of miRNA biogenesis and processing, targeting, and target regulation, see reviews by Brennecke et al. (2005), Brodersen and Voinnet (2009), Huang et al. (2010), and Perron and Provost (2006).

\section{Expression of miRNAs in the embryonic gonad}

Recent studies have identified miRNAs with sexually dimorphic expression patterns in mammalian embryonic gonads, implying that they are active during gonad development (Hossain et al. 2009; Huang et al. 2010; Tripurani et al. 2010; Torley et al. 2011). Though there are no cross-species studies that compare gonadal miRNA expression patterns at comparable time points, there are groups of miRNAs that are commonly up-regulated in the gonads across different species. For example, miRNA 101 (miR-101) is upregulated in the testis of sheep, pig, and mouse (Yu 2005; Luo et al. 2010; Torley et al. 2011). Similarly, miR-202-5p is up-regulated in pig and mouse testes (Mishima et al. 2008; Luo et al. 2010). However, there are studies undermining the importance of miRNAs during embryonic gonadal differentiation, including research on Dicer knockout embryos. Dicer is 


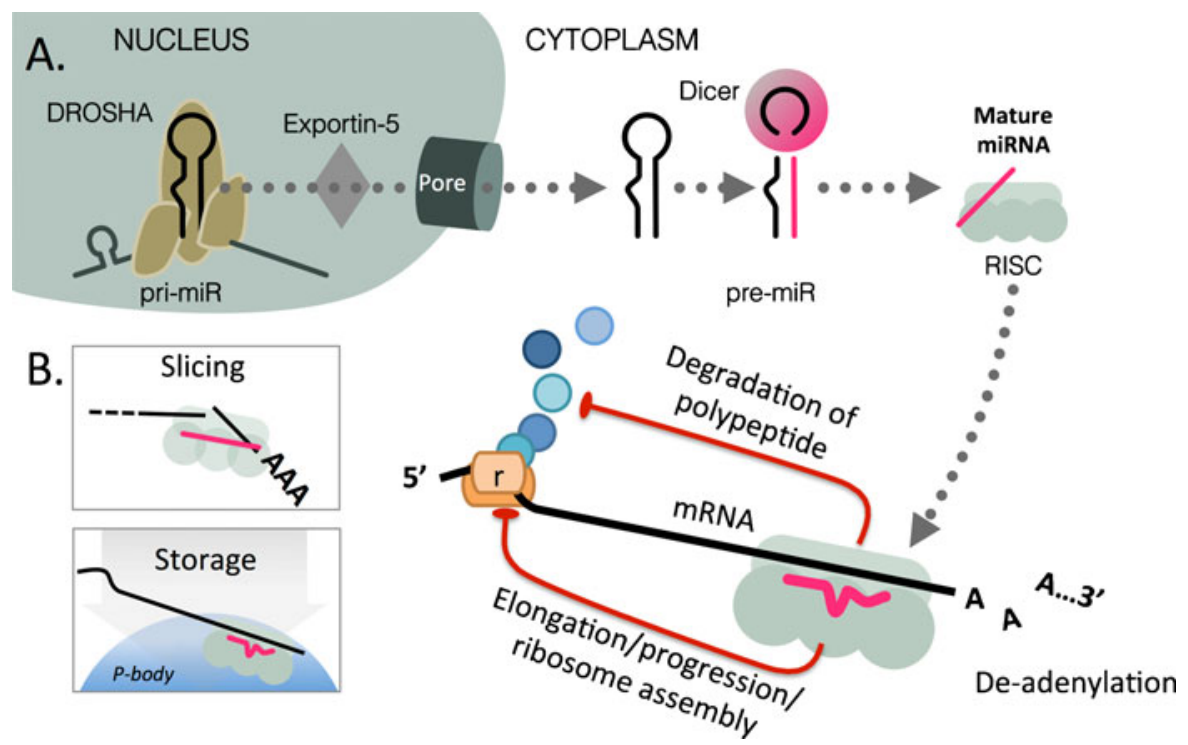

Fig. 3 MicroRNA biogenesis and modes of action. (A) After synthesis, the miRNA forms a secondary hairpin structure that is recognised by Drosha, which cleaves the hairpin from the primary transcript (pri-miR). Exportin-5 exports the hairpin to the cytoplasm, where DICER removes the loop and assists loading of the mature miRNA into the RNA-induced Silencing Complex (RISC). (B) Once loaded into RISC, the miRNA directs RISC to target sites within the target mRNA. RISC usually induces translational silencing by de-adenylation of the mRNA poly A tail, interfereing with polysome formation, degrading the polypeptide as it is synthesised or directly slicing through the miRNA target site. RISC may also direct mRNAs to $\mathrm{p}$ bodies, presumably for future translation or degradation critically required for miRNA maturation, and its knockout results in failure of miRNA biosynthesis. Global Dicer knockout is embryo lethal in mice and zebrafish from E7.0 and E14-15, respectively (Bernstein et al. 2003; Wienholds et al. 2003), implying that miRNAs are indispensible for early embryonic development. Indeed, Dicer and other components of the miRNA machinery are expressed in many different tissues, including testes and ovaries (González-González et al. 2008; Merritt et al. 2008; Pampalakis et al. 2010). To investigate the importance of Dicer in the developing gonad, recent studies have employed gonad-specific conditional Dicer1 knockout mice (Liu et al. 2008; Huang and Yao 2010; Kim et al. 2010; Lei et al. 2010; Papaioannou et al. 2009, 2011). These studies have utilised Cre recombinase driven by gonad-specific or gonad-related promoters, such as the SfI and Amh promoters. In these studies, loss of gonadal Dicer expression in the embryonic gonad causes abnormalities that are only apparent postnatally, such as degeneration of testis cords and arrest of spermatogenesis in males, and retarded folliculogenesis in females (Huang and Yao 2010; Lei et al. 2010). This has led to suggestions that Dicer and miRNAs are not required during embryonic gonadal development. There are, however, some qualifications to this conclusion. For example, Amh-Cre efficiently ablates reporter gene expression in mouse Sertoli cells from E14.0, which may be too late for complete ablation of all miRNAs (Lécureuil et al. 2002; Bingham et al. 2006). Furthermore, miRNAs are still detected at birth (five days later) in the Amh-Cre Dicer knockout Sertoli cells (Papaioannou et al. 2009). These miRNAs were detected at levels only $\sim 2$-fold lower than that of wild-type Sertoli cells. These findings could reflect inefficient or partially penetrant Amh-Cre mediated Dicer knockout. More likely is the robust stability of miRNAs, which prolongs their presence in the absence of Dicer. Prolonged miRNA presence was observed in Dicer $^{--}$zebrafish embryos. MiR-26a was still detected at E8.0 at levels to comparable to Dicer $^{+/-}$ embryos, and also at E14.0, albeit at heavily reduced levels (Wienholds et al. 2003). Furthermore, robust miRNA stability has also been demonstrated after Dicerl knockout in mouse embryonic fibroblasts (Gantier et al. 2011). It is therefore possible that the normal development of Dicer null gonads at embryonic 
stages is due to the stability or sequestration of miRNAs that pre-date Dicer deletion. Lastly, it is also possible that other (redundant) enzymes could mediate miRNA processing in the absence of Dicer.

MiRNAs have been shown to play a role in the development of reproductive tissues and cell types at postnatal stages, for example in the reproductive tract and in germ cells (Hong et al. 2008; Maatouk et al. 2008; Nagaraja et al. 2008; Papaioannou et al. 2011). However, there have been few studies focussing specifically on the embryonic gonads (Ro et al. 2007; Bannister et al. 2009; Huang et al. 2010; Torley et al. 2011; Xu et al. 2011). We recently identified sexually dimorphic miRNAs expressed during embryonic gonadal differentiation in the chicken (Bannister et al. 2009) (Fig. 4). The total number of gonadal miRNAs

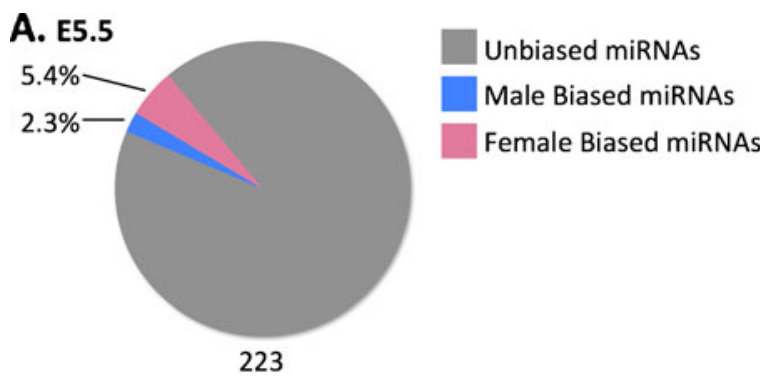

B. E6.5
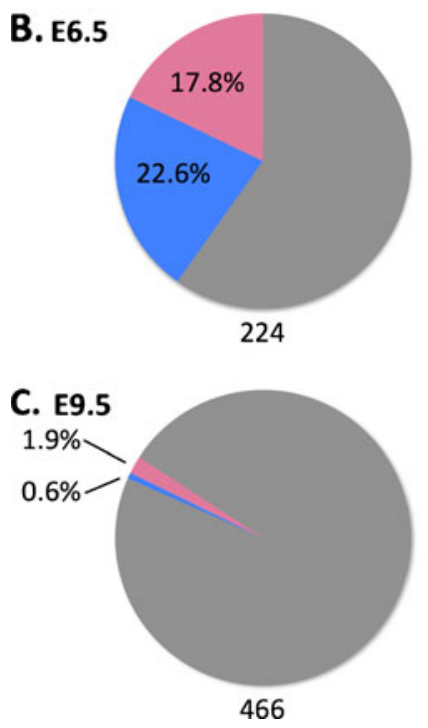

Fig. 4 Total number of chicken gonadal miRNAs and those showing sex-biased expression, as detected by deep sequencing, at stages E5.5, E6.5 and E9.5. Percentages represent miRNAs in the gonads of males (blue) or females (pink) with greater than twofold expression relative to the opposite sex detected from E5.5 to E6.5 was 224. This included a number of miRNAs with male or female biased expression (Fig. 4). By E9.5, 466 gonadal miRNAs were identified; however, fewer showed a sex bias than at earlier stages. This suggests that miRNAs may be performing sex-specific roles earlier rather than later during sexual development. To further investigate the potential role of these miRNAs during gonadal differentiation, we performed qRT-PCR to validate the expression pattern of sex-biased miRNAs (Fig. 5). Gallus gallus (gga) miR-101 was more highly expressed in male compared to female gonads at most time points, although this was not statistically significant (Fig. 5a). However, in females, expression was significantly upregulated between E6.5 and E9.5. Gga-miR-202-5p (miR-202*/miR-202-5p) was significantly up-regulated in males to over 7.5-fold that of E5.5 levels by E9.5, and was specifically male-biased at E9.5 (Fig. 5b). In contrast, gonadal gga-miR-31 was initially significantly male-biased, but relative expression declined during development to become un-biased by E9.5 (Fig. 5c). These results show that miR-202-5p and miR-101 are significantly up-regulated in males and females, respectively, and that miR-31 is significantly downregulated in males after the point of differentiation. Hence, expression profiling suggests that miRNAs participate in gonadal sex differentiation in the chicken model.

\section{Potential roles for miRNAs during embryonic gonad development in the chicken}

Most studies of gonadal miRNAs have focussed on germ cell differentiation and maturation (Toloubeydokhti et al. 2008; He et al. 2009; Papaioannou and Nef 2010). However, one recent study examined miR-363 in the embryonic chicken gonad (Huang et al. 2010). Expression is biphasic in females; high at E3, lower at E4, and then high again at E5. As yet, no target has been identified for miR-363 in the chicken, or in mammals, with which it shares complete homology (Huang et al. 2010). Of the few functional analyses of gonadal miRNAs that have been reported, most have focused on postnatal mammalian tissues. Xu et al. (2011) recently showed that miR-378 regulates aromatase expression, and therefore oestrogen synthesis, in a pre-pubertal porcine granulosa cell line. They suggested that miR-378 might function to restrict aromatase expression to small follicles and prevent interference with oocyte growth. This 

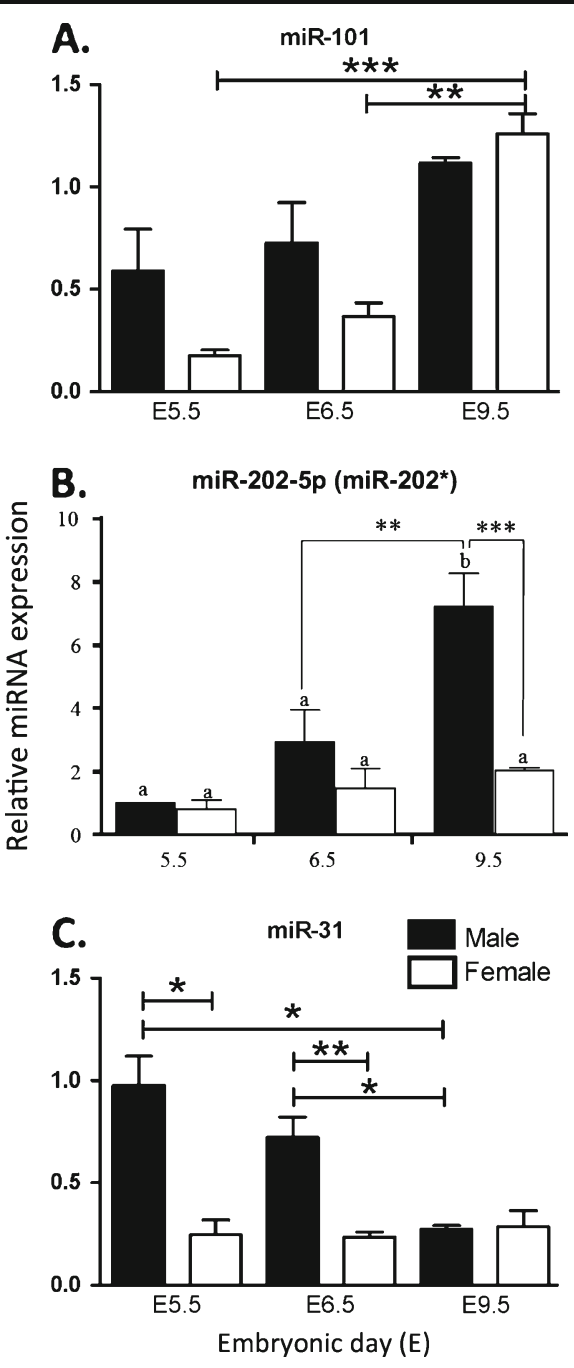

Fig. 5 Relative expression of gonadal miRNAs as detected by qRT-PCR. ( $A$ ) MiR-101 is expressed higher in males at E5.5 and E6.5; however, expression is highly significantly upregulated in females between E5.5 and E9.5 (***p<0.001). (B) miR-202-5p expression is higher in males at E6.5 and E9.5 and increases significantly between E6.5 and E9.5 $(* * p<0.01)$, and is highly male specific at E9.5 $(* * * p<0.001)$. (C) MiR-31 expression is significantly male-biased in males at E5.5 $(* p<0.05)$ and E6.5 $(p<0.01)$ but expression down-regulates from E5.5 to and shows un-biased expression by E9.5. Expression of each miRNA was analysed in triplicate, normalised to U6 reference control, and calculated as fold difference relative to male E5.5 levels. Error bars show standard error of the mean. MiR-202-5p expression data from Bannister et al. (2011), used here with permission

is however the only gonadal miRNA-mRNA interaction that has been clearly demonstrated. In the chicken embryo, we have shown that miR-202-5p is expressed with a male bias during embryonic gonad development
(Bannister et al. 2009, 2011). MiR-202-5p expression is reduced in male and female embryos treated with oestrogen (estradiol-17 $\beta$ ) and increased in female embryos treated with oestrogen inhibitors (Bannister et al. 2011). This suggests that miR-202-5p may play a role downstream of oestrogen signalling, perhaps in maintaining the testis phenotype. However, a gonadal mRNA target for miR-202-5p has yet to be demonstrated.

MiR-101 and miR-31 also showed significant changes in expression during chicken gonad sex differentiation. MiR-101, miR-31 and miR-202-5p are predicted to target components of the TGF- $\beta$ signalling pathway (of which AMH is a member) (Fig. 6). TGF$\beta$ signalling plays a critical role during gonad development (Drummond 2005; Fan et al. 2011), for example, AMH signalling, which is required for Müllerian duct regression (Josso et al. 2005). TGF- $\beta$ signalling involves the binding of a ligand to type II receptors (RII) at the cell surface and subsequent phosphorylation and activation of a type I receptor (RI). Intracellular signalling continues with recruitment and activation of SMAD proteins, which then regulate gene expression by binding to target sequences (Mehra and Wrana 2002). SMAD mediated transcription is regulated by ZEB1 and ZEB2, which promote or inhibit SMAD activity, respectively (Postigo 2003), and TGIF, which represses TGF- $\beta$ responsive genes at the SMAD DNA binding site. In mammals, AMH specifically binds to the AMH RII (AMHR2); however, the RI is likely recruited from other TGF- $\beta$ pathways, such as activin receptors 2 and 3 (ACVR1) (Alk2) and bone morphogenic protein receptor 1 (BMPR1A) (Alk3) (Jamin et al. 2003; Belville et al. 2005). BMPR1B, another TGF- $\beta$ receptor, also binds $\mathrm{AMH}$, but has a competitive inhibitory influence on AMH signalling. In the mammalian gonad, AMHR2 is expressed by Leydig and Sertoli cells in males (Racine et al. 1998; Salhi et al. 2004) and granulosa cells in females (Josso et al. 2001). It therefore is likely to have embryonic roles beyond Müllerian duct regression. However, to date, avian AMHR2 remains undefined.

Interestingly, miR-101 is predicted to target TGIF1, ZEB2 and BMPR1B (TargetScan, Lewis et al. 2005), which inhibit TGF- $\beta$ signalling. Therefore, miR-101 potentially inhibits the repressive effects of TGIF1, ZEB2 and BMPR1B during TGF- $\beta$ and AMH signalling. MiR-101 is also predicted to target ZEB1, which promotes SMAD transduction of TGF- $\beta$ signals to gene targets. In male gonads, miR-101 may therefore 


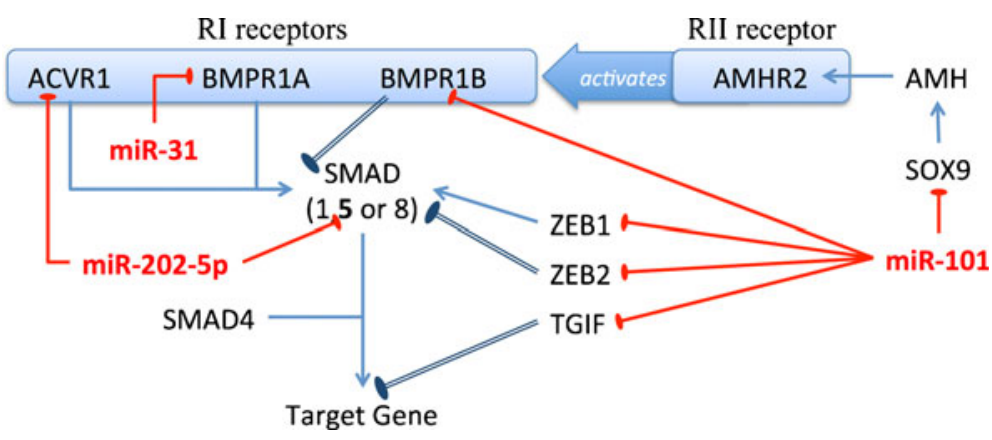

Fig. 6 AMH signal transduction via the TGF- $\beta$ signalling pathway and potential involvement of miRNAs. AMH binds specifically to the AMH receptor (AMHR2), which activates either activin receptor 1 (ACVR1), or bone morphogenic receptor 1A (BMPR1A), or 1B (BMPR1B). Activated ACVR1 and BMPR1A transduce AMH signals by activating SMAD signalling proteins (SMAD1, 5 or 8), with assistance from ZEB1. BMPR1B competitively antagonises SMAD activation. ZEB2 and TGIF bind to SMADs and SMAD DNA binding sites, respectively, to inhibit signalling. MiR-101 (red) is predicted to target $B M P R 1 B, Z E B 1$ and 2, and $T G I F$ transcripts, and may modulate TGF- $\beta$ signalling. miR-202-5p and miR-31 (red) are predicted to target $A C V R 1$ and SMAD5, and BMPR1A transcripts, respectively, and may assist a shifting TGF- $\beta$ signalling pathways in males post-gonadal sex differentiation

regulates spermatogonial differentiation in postnatal testis (Pellegrini et al. 2003), which may be regulated by miR-202-5p repression of 'competing' RIs, such as ACVR1, and modulation of SMAD5.

A highly conserved binding site for miR-101 is also predicted in the 3' UTR of SOX9 (TargetScan, Lewis et al. 2005; Torley et al. 2011). Our results show that miR101 is more highly expressed in males but increases significantly in females after gonadal differentiation (E9.5; Fig. 5a). This suggests that miR-101 may act to reinforce the identity of ovarian cell types once differentiation has occurred, perhaps by suppressing aberrant SOX9 expression. In males, miR-101 may act to modulate or fine tune SOX9 expression, or prevent aberrant SOX9 expression in the wrong cell type. The exact cell type expressing miR-101 in the embryonic chicken gonad is presently unknown. If miR-101 co-localises with $S O X 9$ in the developing Sertoli cells of males for example, it may a have a role in regulating SOX9 expression. It would also be interesting to determine if miR-101 localises to granulosa cells in the ovary and if its expression is altered in FOXL2 or RSPO1 null animals.

\section{Validating and functionally analysing gonadal miRNAs}

Recent screens have highlighted miRNAs as potential regulators of gonadal development. Although one miRNA, miR-378, has been found to regulate oestrogen 
synthesis in the porcine ovary (Xu et al. 2011), targets of the many other gonadal miRNAs remain unknown. Demonstration of a bona-fide miRNA-mRNA relationship is challenging and requires approaches from many angles, including identification of mRNA targets in vivo. A miRNA must be present at the same time and in the same cell as its target transcript for regulation to occur (although there is emerging evidence for miRNAs being exported from the cell; see Gursanscky et al. 2011). Therefore, expression patterns for the miRNA, its predicted target transcript, and the protein need to be well characterised. To this end, we are currently comparing next generation sequencing data sets for chicken gonadal miRNAs with gonadal mRNAs. Alternative methods of validating next generation miRNA sequencing include Northern blots and whole-mount in situ hybridisation (WISH) to detect miRNA and mRNA transcripts, and Western and immunostaining to detect protein levels. Northern versus Western blotting of target genes may clarify if a given mRNA is regulated by translational inhibition. WISH data can complement blot data, and determine if expression of miRNAs and potential targets spatially overlap within a tissue.

A number of biochemical approaches have recently been developed to identify miRNA targets on a more global scale (Brennecke et al. 2005; Karginov et al. 2007; Bartel 2009). For example, cross-linking immunoprecipitation (CLIP) involves isolating and crosslinking miRNA-RISC complexes followed by immunoprecipitation and high throughput sequencing (Chi et al. 2009; Zisoulis et al. 2011). MiRNA-mRNA interactions can also be identified using "pull-down" type assays. For example, biotinylated miRNAs can be transfected into appropriate cell cultures to hybridise to endogeneous mRNA targets and then captured via streptavidin solid phase (Ørom and Lund 2007). RNA can then be purified and analysed to identify the targets. Another example involves pulling-down miRISC complexes by immuno-purification of FLAG-tagged RISC (Easow et al. 2007). Both miRNAs and mRNAs can be identified by microarray or sequencing, and potential targets can be determined by matching enriched miRNAs sequences to enriched miRNA binding sites present in the isolated mRNAs (Easow et al. 2007).

These methods may show a close association between individual miRNAs and potential mRNA targets; however, they do not prove functional interactions. Luciferase reporter assays have been widely used to demonstrate specific miRNA interaction with predicted target transcripts (Lee et al. 2006; Easow et al. 2007; Li et al. 2007; Lytle et al. 2007; Lin et al. 2008; Takada et al. 2009; Xu et al. 2011). In these experiments, the 3' UTR of putative target genes are cloned downstream of a luciferase reporter and expressed in the presence of potential regulatory miRNAs. A functional interaction between the $3^{\prime}$ UTR and the co-expressed miRNA is expected to reduce luciferase expression. We are currently testing chicken TGF- $\beta$ 3'UTRs for regulation by miRNAs using this method.

The exact role of miRNAs, if any, during gonadal sex differentiation is still unknown. In vivo modulation of miRNA expression is likely to give the clearest insight into function, irrespective of target mRNA predictions. We are currently using the RCAS chicken retroviral vector to over-express endogenous miRNAs or synthetic siRNAs, using a Pol III (U6) promoter (Smith et al. 2009). Alternatively, we are exploring the use of so-called molecular sponges, which act as decoys to bind endogenous miRNAs in a sequence specific manner and effectively knock down their function (Ebert et al. 2007; Ebert and Sharp 2010). Theoretically, such sponge sequences could be delivered to developing chicken embryos using the RCAS viral vector. Another approach involves the use of antagomirs to knockdown miRNA function in ovo (Krützfeldt et al. 2005, 2007). This approach has been used to knockdown the function of miR-196 in ovo, revealing a role for this miRNA in patterning the axial skeleton in chicken embryos (McGlinn et al. 2009).

\section{Conclusion}

MiRNAs are important regulators of gene expression in animals, playing roles in cell fate decisions, development, and disease. However, our understanding of gonadal miRNAs and their role during sexual differentiation is rudimentary. Expression profiling suggests that miRNAs have a function in embryonic gonadal development. In the chicken gonad, a large number of miRNAs are expressed, some of which show sexually dimorphic expression patterns during differentiation. We have found that miR-101, miR-31 and miR-202-5p show expression patterns strongly indicating a role in gonadal sexual differentiation. Functional analysis will define the precise roles for these miRNAs in testicular and ovarian development. 
Acknowledgements We acknowledge the Victorian Government's Operational Infrastructure Support Program, the National Health and Medical Research Council and the Poultry Cooperative Research Centre who supported the research presented in this paper. We thank the reviewers of this article for their constructive feedback.

\section{References}

Bannister SC, Tizard MLV, Doran TJ et al (2009) Sexually dimorphic microRNA expression during chicken embryonic gonadal development. Biol Reprod 81:165-176

Bannister SC, Smith CA, Roeszler KN et al (2011) Manipulation of estrogen synthesis alters MIR202* expression in embryonic chicken gonads. Biol Reprod 85:22-30

Barske LA, Capel B (2008) Blurring the edges in vertebrate sex determination. Curr Opin Genet Dev 18:499-505

Bartel DP (2009) MicroRNAs: target recognition and regulatory functions. Cell 136:215-233

Belville C, Jamin SP, Picard J-Y et al (2005) Role of type I receptors for anti-Mullerian hormone in the SMAT-1 Sertoli cell line. Oncogene 24:4984-4992

Bernstein E, Kim SY, Carmell MA et al (2003) Dicer is essential for mouse development. Nat Genet 35:215-217

Bingham NC, Verma-Kurvari S, Parada LF, Parker KL (2006) Development of a steroidogenic factor $1 /$ Cre transgenic mouse line. Genesis 44:419-424

Blount AL, Schmidt K, Justice NJ et al (2009) Foxl2 and Smad3 coordinately regulate follistatin gene transcription. J Biol Chem 284:7631-7645

Brennecke J, Stark A, Russell RB, Cohen SM (2005) Principles of microRNA - target recognition. PLoS Biol 3:e85

Brodersen P, Voinnet O (2009) Revisiting the principles of microRNA target recognition and mode of action. Nat Rev Mol Cell Biol 10:141-148

Chi SW, Zang JB, Mele A, Darnell RB (2009) Argonaute HITSCLIP decodes microRNA-mRNA interaction maps. Nature 460:479-486

Chue J, Smith CA (2011) Sex determination and sexual differentiation in the avian model. FEBS J 278:1027-1034

De Santa BP, Bonneaud N, Boizet B et al (1998) Direct interaction of SRY-related protein SOX9 and steroidogenic factor 1 regulates transcription of the human anti-Müllerian hormone gene. Mol Cell Biol 18:6653-6665

DeFalco T, Capel B (2009) Gonad morphogenesis in vertebrates: divergent means to a convergent end. Annu Rev Cell Dev Biol 25:457-482

Drummond AE (2005) TGF $\beta$ signalling in the development of ovarian function. Cell Tissue Res 322:107-115

Duursma AM, Kedde M, Schrier M et al (2008) miR-148 targets human DNMT3b protein coding region. RNA 14:872-877

Easow G, Teleman AA, Cohen SM (2007) Isolation of microRNA targets by miRNP immunopurification. RNA 13:1198-1204

Ebert MS, Sharp PA (2010) MicroRNA sponges: progress and possibilities. RNA 16:2043-2050

Ebert MS, Neilson JR, Sharp PA (2007) MicroRNA sponges: competitive inhibitors of small RNAs in mammalian cells. Nat Meth 4:721-726
Elbrecht A, Smith R (1992) Aromatase enzyme activity and sex determination in chickens. Science 255:467-470

Fan Y-S, Hu Y-J, Yang W-X (2011) TGF- $\beta$ superfamily: how does it regulate testis development. Mol Biol Rep (in press)

Fleming NI, Knower KC, Lazarus KA et al (2010) Aromatase is a direct target of FOXL2: C134W in granulosa cell tumors via a single highly conserved binding site in the ovarian specific promoter. PLoS One 5:e14389

Forman JJ, Legesse-Miller A, Coller HA (2008) A search for conserved sequences in coding regions reveals that the let7 microRNA targets Dicer within its coding sequence. Proc Natl Acad Sci USA 105:14879-14884

Frankenhuis MT, Kappert HJ (1980) Experimental transformation of right gonads of female fowl in to fertile testes. Biol Reprod 23:526-529

Gantier MP, McCoy CE, Rusinova I et al (2011) Analysis of microRNA turnover in mammalian cells following Dicer1 ablation. Nucleic Acids Res 39:5692-5703

Gigli I, Cushman RA, Wahl CM, Fortune JE (2005) Evidence for a role for anti-Müllerian hormone in the suppression of follicle activation in mouse ovaries and bovine ovarian cortex grafted beneath the chick chorioallantoic membrane. Mol Reprod Dev 71:480-488

González-González E, López-Casas PP, del Mazo J (2008) The expression patterns of genes involved in the RNAi pathways are tissue-dependent and differ in the germ and somatic cells of mouse testis. Biochim Biophys Acta 1779:306-311

Govoroun MS, Pannetier M, Pailhoux E et al (2004) Isolation of chicken homolog of the FOXL2 gene and comparison of its expression patterns with those of aromatase during ovarian development. Dev Dyn 231:859-870

Graves JA (2009) Sex determination: birds do it with a $\mathrm{Z}$ gene. Nature 461:177-178

Gursanscky NR, Searle IR, Carroll BJ (2011) Mobile microRNAs hit the target. Traffic 12:1475-1482

Hamburger V, Hamilton HL (1951) A series of normal stages in the development of the chick embryo. J Morph 88:49-92

Harris SE, Chand AL, Winship IM et al (2002) Identification of novel mutations in FOXL2 associated with premature ovarian failure. Mol Hum Reprod 8:729-733

He Z, Kokkinaki M, Pant D et al (2009) Small RNA molecules in the regulation of spermatogenesis. Reproduction 137:901-911

Hong X, Luense LJ, McGinnis LK et al (2008) Dicer1 is essential for female fertility and normal development of the female reproductive system. Endocrinology 149:6207-6212

Hossain M, Ghanem N, Hoelker M et al (2009) Identification and characterization of miRNAs expressed in the bovine ovary. BMC Genomics 10:443

Huang C-CJ, Yao HH (2010) Inactivation of Dicer1 in steroidogenic factor 1-positive cells reveals tissue-specific requirement for Dicer1 in adrenal, testis, and ovary. BMC Dev Biol 10:66-66

Huang P, Gong Y, Peng X et al (2010) Cloning, identification, and expression analysis at the stage of gonadal sex differentiation of chicken miR-363 and 363. Acta Biochim Biophys Sin 42:522-529

Hudson QJ, Smith CA, Sinclair AH (2005) Aromatase inhibition reduces expression of FOXL2 in the embryonic chicken ovary. Dev Dyn 233:1052-1055

Jamin SP, Arango NA, Mishina Y et al (2003) Genetic studies of the AMH/MIS signaling pathway for Müllerian duct regression. Mol Cell Endocrinol 211:15-19 
Josso N, di Clemente N, Gouédard L (2001) Anti-Mullerian hormone and its receptors. Mol Cell Endocrinol 179:25-32

Josso N, Belville C, di Clemente N, Picard J-Y (2005) AMH and $\mathrm{AMH}$ receptor defects in persistent Müllerian duct syndrome. Hum Reprod Update 11:351-356

Karginov FV, Conaco C, Xuan Z et al (2007) A biochemical approach to identifying microRNA targets. Proc Natl Acad Sci USA 104:19291-19296

Kim Y, Kobayashi A, Sekido R et al (2006) Fgf9 and Wnt4 act as antagonistic signals to regulate mammalian sex determination. PLoS Biol 4(6):e187

Kim G-J, Georg I, Scherthan H et al (2010) Dicer is required for Sertoli cell function and survival. Int J Dev Biol 54:867-875

Knight PG, Glister C (2006) TGF- $\beta$ superfamily members and ovarian follicle development. Reproduction 132:191-206

Koba N, Ohfuji T, Ha Y et al (2008) Profiles of mRNA expression of FOXL2, P450arom, DMRT1, AMH, P450c17, SF1, $\mathrm{ER} \alpha$ and $\mathrm{AR}$, in relation to gonadal sex differentiation in duck embryo. J Poult Sci 45:132-138

Kobayashi A (2005) Sox9 in testis determination. Ann NY Acad Sci 1061:9-17

Koopman P (2001) The genetics and biology of vertebrate sex determination. Cell 105:843-847

Krützfeldt J, Rajewsky N, Braich R et al (2005) Silencing of microRNAs in vivo with 'antagomirs'. Nature 438:685689

Krützfeldt J, Kuwajima S, Braich R et al (2007) Specificity, duplex degradation and subcellular localization of antagomirs. Nucleic Acids Res 35:2885-2892

Lasala C, Schteingart HF, Arouche N et al (2011) SOX9 and SF1 are involved in cyclic AMP-mediated upregulation of antiMüllerian gene expression in the testicular prepubertal Sertoli cell line SMAT1. Am J Physiol-Endoc M 301:E539-E547

Lécureuil C, Fontaine I, Crepieux P, Guillou F (2002) Sertoli and granulosa cell-specific Cre recombinase activity in transgenic mice. Genesis 33:114-118

Lee H-J, Palkovits M, Young WS (2006) miR-7b, a microRNA up-regulated in the hypothalamus after chronic hyperosmolar stimulation, inhibits Fos translation. Proc Natl Acad Sci USA 103:15669-15674

Lei L, Jin S, Gonzalez G et al (2010) The regulatory role of Dicer in folliculogenesis in mice. Mol Cell Endocrinol 315:63-73

Lewis BP, Burge CB, Bartel DP (2005) Conserved seed pairing, often flanked by adenosines, indicates that thousands of human genes are microRNA targets. Cell 120:15-20

Li Q-J, Chau J, Ebert PJR et al (2007) miR-181a is an intrinsic modulator of T cell sensitivity and selection. Cell 129:147161

Lin CJ-F, Gong H-Y, Tseng H-C et al (2008) miR-122 targets an anti-apoptotic gene, Bcl-w, in human hepatocellular carcinoma cell lines. Biochem Biophys Res Commun 375:315320

Liu J, Rivas FV, Wohlschlegel J et al (2005) A role for the Pbody component GW182 in microRNA function. Nat Cell Biol 7:1261-1266

Liu H-C, Tang Y, He Z et al (2008) Dicer and miRNA, key players in oogenesis. Fertil Steril 90:S81-S82

Liu C-F, Bingham N, Parker K, Yao HH-C (2009) Sex-specific roles of $\beta$-catenin in mouse gonadal development. Hum Mol Genet 18:405-417
Lovell-Badge R, Canning C, Sekido R (2002) Sex-determining genes in mice: building pathways. Novartis Found Symp 244:4-18; discussion 18-22, 35-42, 253-257

Luo L, Ye L, Liu G et al (2010) Microarray-based approach identifies differentially expressed microRNAs in porcine sexually immature and mature testes. PLoS One 18;5(8): e11744

Lytle JR, Yario TA, Steitz JA (2007) Target mRNAs are repressed as efficiently by microRNA-binding sites in the $5^{\prime}$ UTR as in the 3' UTR. Proc Natl Acad Sci USA 104:9667-9672

Maatouk DM, Loveland KL, McManus MT et al (2008) Dicer1 is required for differentiation of the mouse male germline. Biol Reprod 79:696-703

MacLaughlin DT, Donahoe PK (2004) Sex determination and differentiation. N Engl J Med 350:367-378

Maraud R, Vergnaud O, Rashedi M (1990) New insights on the mechanism of testis differentiation from the morphogenesis of experimentally induced testes in genetically female chick embryos. Am J Anat 188:429-437

Martineau J, Nordqvist K, Tilmann C et al (1997) Male-specific cell migration into the developing gonad. Curr Biol 7:958968

Matson CK, Murphy MW, Sarver AL et al (2011) DMRT1 prevents female reprogramming in the postnatal mammalian testis. Nature 476:101-104

McGlinn E, Yekta S, Mansfield JH et al (2009) In ovo application of antagomiRs indicates a role for miR-196 in patterning the chick axial skeleton through Hox gene regulation. Proc Natl Acad Sci USA 106:18610-18615

Mehra A, Wrana JL (2002) TGF- $\beta$ and the Smad signal transduction pathway. Biochem Cell Biol 80:605-622

Merritt WM, Lin YG, Han LY et al (2008) Dicer, Drosha, and outcomes in patients with ovarian cancer. N Engl J Med 359:2641-2650

Mishima T, Takizawa T, Luo S-S et al (2008) MicroRNA (miRNA) cloning analysis reveals sex differences in miRNA expression profiles between adult mouse testis and ovary. Reproduction 136:811-822

Morrish B, Sinclair A (2002) Vertebrate sex determination: many means to an end. Reproduction 124:447-457

Nagaraja AK, Andreu-Vieyra C, Franco HL et al (2008) Deletion of Dicer in somatic cells of the female reproductive tract causes sterility. Mol Endocrinol 22:2336-2352

Oreal E, Pieau C, Mattei M-G et al (1998) Early expression of $\mathrm{AMH}$ in chicken embryonic gonads precedes testicular SOX9 expression. Dev Dyn 212:522-532

Oreal E, Mazaud S, Picard J-Y et al (2002) Different patterns of anti-Mullerian hormone expression, as related to DMRT1, SF-1, WT1, GATA-4, Wnt-4, and Lhx9 expression, in the chick differentiating gonads. Dev Dyn 225:221-232

Ørom UA, Lund AH (2007) Isolation of microRNA targets using biotinylated synthetic microRNAs. Methods 43: $162-165$

Pampalakis G, Diamandis EP, Katsaros D, Sotiropoulou G (2010) Down-regulation of dicer expression in ovarian cancer tissues. Clin Biochem 43:324-327

Papaioannou MD, Nef S (2010) microRNAs in the testis: building up male fertility. J Androl 31:26-33

Papaioannou MD, Pitetti J-L, Ro S et al (2009) Sertoli cell Dicer is essential for spermatogenesis in mice. Dev Biol 326:250 259 
Papaioannou MD, Lagarrigue M, Vejnar CE et al (2011) Loss of Dicer in Sertoli cells has a major impact on the testicular proteome of mice. Mol Cell Proteimucs 10(4):M900587MCP200

Pauli A, Rinn JL, Schier AF (2011) Non-coding RNAs as regulators of embryogenesis. Nat Rev Genet 12:136-149

Pellegrini M, Grimaldi P, Rossi P et al (2003) Developmental expression of BMP4/ALK3/SMAD5 signaling pathway in the mouse testis: a potential role of BMP4 in spermatogonia differentiation. J Cell Sci 116:3363-3372

Perron MP, Provost P (2006) Protein interactions and complexes in human microRNA biogenesis and function. Front Biosci $13: 2537-2547$

Postigo AA (2003) Opposing functions of ZEB proteins in the regulation of the TGF $\beta / B M P$ signaling pathway. EMBO J 22:2443-2452

Racine C, Rey R, Forest MG et al (1998) Receptors for antiMüllerian hormone on Leydig cells are responsible for its effects on steroidogenesis and cell differentiation. Proc Natl Acad Sci USA 95:594-599

Rashedi M, Maraud R, Piet M et al (1990) Influence of heterospecific testis graft on the gonadal sex differentiation of female bird embryos. Cell Differ Dev 32:167-174

Rey R, Lukas-Croisier C, Lasala C, Bedecarrás P (2003) AMH/ MIS: what we know already about the gene, the protein and its regulation. Mol Cell Endocrinol 211:21-31

Ro S, Park C, Young D et al (2007) Tissue-dependent paired expression of miRNAs. Nucleic Acids Res 35:5944-5953

Salhi I, Cambon-Roques S, Lamarre I et al (2004) The antiMüllerian hormone type II receptor: insights into the binding domains recognized by a monoclonal antibody and the natural ligand. Biochem J 379:785-793

Santibañez JF, Quintanilla M, Bernabeu C (2011) TGF- $\beta$ /TGF$\beta$ receptor system and its role in physiological and pathological conditions. Clin Sci (Lond) 121:233-251

Scheib D (1983) Effects and role of estrogens in avian gonadal differentiation. Differentiation 23(Suppl):S87-S92

Schmidt D, Ovitt CE, Anlag K et al (2004) The murine wingedhelix transcription factor Fox12 is required for granulosa cell differentiation and ovary maintenance. Development 131:933-942

Sekido R (2010) SRY: a transcriptional activator of mammalian testis determination. Int J Biochem Cell Biol 42:417-420

Sekido R, Lovell-Badge R (2007) Mechanisms of gonadal morphogenesis are not conserved between chick and mouse. Dev Biol 302:132-142

Sekido R, Lovell-Badge R (2009) Sex determination and SRY: down to a wink and a nudge? Trends Genet 25:19-29

Sinclair A, Smith C (2009) Females battle to suppress their inner male. Cell 139:1051-1053

Smith CA (2007) Sex determination in birds: HINTs from the W sex chromosome? Sex Dev 1:279-285

Smith CA (2010) Sex determination in birds: a review. EMU 110:364

Smith CA, Sinclair AH (2004) Sex determination: insights from the chicken. Bioessays 26:120-132

Smith CA, Katz M, Sinclair AH (2003) Dmrt1 is upregulated in the gonads during female-to-male sex reversal in $\mathrm{ZW}$ chicken embryos. Biol Reprod 68:560-570
Smith CA, McClive PJ, Hudson Q, Sinclair AH (2005) Malespecific cell migration into the developing gonad is a conserved process involving PDGF signalling. Dev Biol 284:337-350

Smith CA, Roeszler KN, Ohnesorg T et al (2009) The avian Z-linked gene DMRT1 is required for male sex determination in the chicken. Nature 461:267-271

Suh N, Blelloch R (2011) Small RNAs in early mammalian development: from gametes to gastrulation. Development 138:1653-1661

Takada S, Berezikov E, Choi YL et al (2009) Potential role of miR-29b in modulation of Dnmt3a and Dnmt3b expression in primordial germ cells of female mouse embryos. RNA 15:1507-1514

Toloubeydokhti T, Bukulmez O, Chegini N (2008) Potential regulatory functions of microRNAs in the ovary. Semin Reprod Med 26:469-478

Tomizuka K, Horikoshi K, Kitada R et al (2008) R-spondin1 plays an essential role in ovarian development through positively regulating Wnt-4 signaling. Hum Mol Gen 17:1278-1291

Torley KJ, da Silveira JC, Smith P et al (2011) Expression of miRNAs in ovine fetal gonads: potential role in gonadal differentiation. Reprod Biol Endocrinol 9:2

Tripurani SK, Xiao C, Salem M, Yao J (2010) Cloning and analysis of fetal ovary microRNAs in cattle. Anim Reprod Sci 120:16-22

Uhlenhaut NH, Jakob S, Anlag K et al (2009) Somatic sex reprogramming of adult ovaries to testes by FOXL2 ablation. Cell 139:1130-1142

Vaillant S, Magre S, Dorizzi M et al (2001) Expression of AMH, SF1, and SOX9 in gonads of genetic female chickens during sex reversal induced by an aromatase inhibitor. Dev Dyn 222:228-237

Vasudevan S, Tong Y, Steitz JA (2007) Switching from repression to activation: microRNAs can up-regulate translation. Science 318:1931-1934

Veitia RA (2010) FOXL2 versus SOX9: a lifelong 'battle of the sexes'. BioEssays 32:375-380

Wienholds E, Koudijs MJ, van Eeden FJM et al (2003) The microRNA-producing enzyme Dicer1 is essential for zebrafish development. Nat Genet 35:217-218

Wilhelm D, Palmer S, Koopman P (2007) Sex determination and gonadal development in mammals. Physiol Rev 87:128

$\mathrm{Xu} \mathrm{S}$, Linher-Melville K, Yang BB et al (2011) MicroRNA378 (miR-378) regulates ovarian estradiol production by targeting aromatase. Endocrinology 152:39413951

Yu Z (2005) MicroRNA Mirn122a reduces expression of the posttranscriptionally regulated germ cell transition protein 2 (Tnp2) messenger RNA (mRNA) by mRNA cleavage. Biol Reprod 73:427-433

Zhao D, McBride D, Nandi S et al (2010) Somatic sex identity is cell autonomous in the chicken. Nature 464:237-242

Zisoulis DG, Yeo GW, Pasquinelli AE (2011) Comprehensive identification of miRNA target sites in live animals. In: Dalmay T (eds) MicroRNAs in Development. Humana Press, pp 169-185 